\title{
Erratum: Universal Relations for Gravitational-Wave Asteroseismology of Protoneutron Stars \\ [Phys. Rev. Lett. 123, 051102 (2019)]
}

Alejandro Torres-Forné $\odot$, Pablo Cerdá-Durán, Martin Obergaulinger, Bernhard Müller, and José A. Font

(Received 8 November 2021; published 3 December 2021)

DOI: 10.1103/PhysRevLett.127.239901

There is a factor 2 missing in the calculation of the ratio $r \equiv M_{\mathrm{PNS}} / R_{\mathrm{PNS}}^{2}$ in this Letter. Additionally, not all the data from the simulations with CoCoNuT were used for the fits and for the plots. As a result, there is a constant factor difference in Fig. 2 (a factor $\sqrt{2}$ on the left panel and 2 in the right panel affecting only the ordinate) and the coefficients in Table I have to be modified (approximately a factor $1 / 2$ for $b, 1 / 4$ for $c$, and $1 / 8$ for $d$ ). Table I and Fig. 2 presented here replace Table I and Fig. 2 in the Letter, respectively, correcting those factors and adding the missing data.
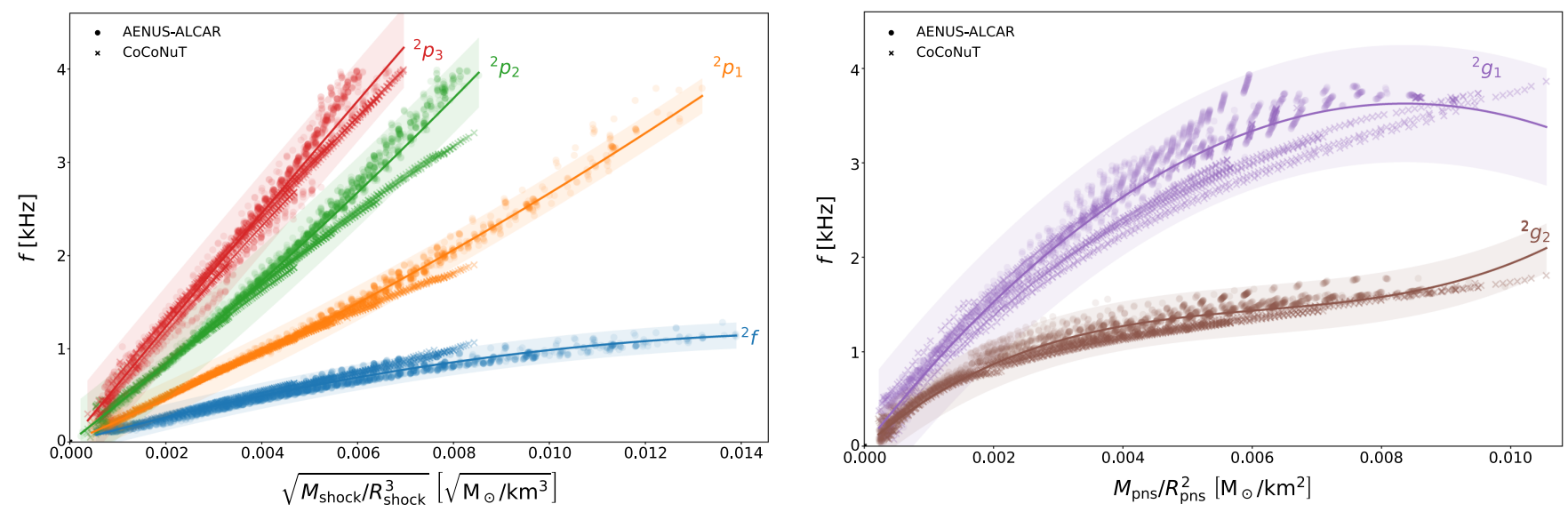

FIG. 2. Fits of the different modes. The left-hand panel shows the $f$ mode and the first three $p$ modes while the right-hand panel shows the first two $g$ modes. The results from AENUS-ALCAR and CoCoNuT are represented with solid circles and crosses, respectively. Shaded areas indicate $2 \sigma$ error intervals.

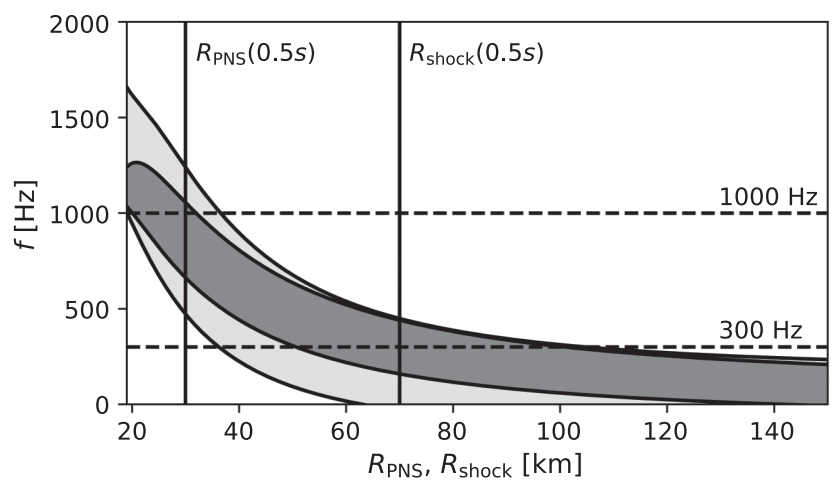

FIG. 3. Range of frequencies for the ${ }^{2} g_{2}$ (light shade) and the ${ }^{2} f$ mode (dark shade) as a function of the PNS and shock radius, respectively, for masses in the interval $1.2 M_{\odot}-2.5 M_{\odot}$ considering $2 \sigma$ errors from the universal relations. Vertical lines show the PNS and shock radius $0.5 \mathrm{~s}$ postbounce and horizontal dashed lines the frequency of the ${ }^{2} g_{2}$ mode $(\sim 1000 \mathrm{~Hz})$ and the ${ }^{2} f$ mode $(\sim 300 \mathrm{~Hz})$ at the same time. 
TABLE I. Parameters of the universal relations found for each of the modes considered. Fits are of the form $f=a+b x+c x^{2}+d x^{3}$, with $f$ expressed in hertz, and using $M_{\odot}$ and kilometers as units for mass and length in the quantities expressed in the variable $x$. Note that some coefficients are not used in some fits. $R^{2}$ and $\sigma$ are the correlation coefficient and the standard deviation of the data (in hertz) with respect to the fit, respectively.

\begin{tabular}{lcccccrr}
\hline \hline Mode & $x$ & $a$ & $b / 10^{5}$ & $c / 10^{6}$ & $d / 10^{9}$ & $R^{2}$ & $\sigma$ \\
\hline${ }^{2} f$ & $\sqrt{M_{\text {shock }} / R_{\text {shock }}^{3}}$ & $\ldots$ & $1.410 \pm 0.004$ & $-4.23 \pm 0.06$ & $\ldots$ & 0.966 & 45 \\
${ }^{2} p_{1}$ & $\sqrt{M_{\text {shock }} / R_{\text {shock }}^{3}}$ & $\ldots$ & $2.205 \pm 0.007$ & $4.63 \pm 0.09$ & $\ldots$ & 0.991 & 61 \\
${ }^{2} p_{2}$ & $\sqrt{M_{\text {shock }} / R_{\text {shock }}^{3}}$ & $\ldots$ & $4.02 \pm 0.02$ & $7.4 \pm 0.3$ & $\ldots$ & 0.983 & 123 \\
${ }^{2} p_{3}$ & $\sqrt{M_{\text {shock }} / R_{\text {shock }}^{3}}$ & $\ldots$ & $6.21 \pm 0.03$ & $-1.9 \pm 0.6$ & $\ldots$ & 0.979 & 142 \\
${ }^{2} g_{1}$ & $M_{\text {PNS }} / R_{\text {PNS }}^{2}$ & $\ldots$ & $8.67 \pm 0.03$ & $-51.9 \pm 0.5$ & $\ldots$ & 0.958 & 205 \\
${ }^{2} g_{2}$ & $M_{\text {PNS }} / R_{\text {PNS }}^{2}$ & $\ldots$ & $5.88 \pm 0.03$ & $-86.2 \pm 1.0$ & $4.67 \pm 0.08$ & 0.956 & 85 \\
${ }^{2} g_{3}$ & $\sqrt{M_{\text {shock }} / R_{\text {shock }}^{3} p_{C} / \rho_{C}^{2.5}}$ & $905 \pm 3$ & $-79.9 \pm 1.7$ & $-11000 \pm 2000$ & $\ldots$ & 0.925 & 41 \\
\hline \hline
\end{tabular}

There was an additional error in the coefficients $b$ and $c$ for the ${ }^{2} g_{3}$ mode in Table I that has been corrected in the new Table I. Some of the labels in the header were misleading and have been improved. We note that the units for $\rho_{C}$ and $p_{C}$ used for the fit in this table are centimeters (using $G=c=1$ ), information that was missing in the previous version of the Letter.

Figure 3 of the Letter has also been modified to use the updated values of the fits. The text has been modified accordingly. The updated measurement of the PNS radius at $0.5 \mathrm{~s}$ is $19-37 \mathrm{~km}$ (instead of 28-52 km), which fits much better the radius measured directly from the simulation $(30 \mathrm{~km}$ ). Similarly, the updated constraint for the shock radius is $50-100 \mathrm{~km}$ (instead of $64-127 \mathrm{~km}$ ) to be compared with $70 \mathrm{~km}$ obtained from the simulation.

These changes leave all the conclusions of the Letter unaffected. 